\title{
Molecular Entrapment
}

National Cancer Institute

\section{Source}

National Cancer Institute. Molecular Entrapment. NCI Thesaurus. Code C81872.

A molecular interaction in which a small molecule or structure becomes trapped, usually in a larger molecule or structure, due to non-covalent interactions. 